\title{
The Work of a Normal Force
}

\author{
Pirooz Mohazzabi \\ Department of Mathematics and Physics, University of Wisconsin-Parkside, Kenosha, WI, USA \\ Email: mohazzab@uwp.edu
}

How to cite this paper: Mohazzabi, P. (2017) The Work of a Normal Force. Journal of Applied Mathematics and Physics, $\mathbf{5}$, 1113-1119.

https://doi.org/10.4236/jamp.2017.55098

Received: March 30, 2017

Accepted: May 23, 2017

Published: May 27, 2017

Copyright $\odot 2017$ by author and Scientific Research Publishing Inc. This work is licensed under the Creative Commons Attribution International License (CC BY 4.0).

http://creativecommons.org/licenses/by/4.0/

\begin{abstract}
It is shown that an impulsive force acting on a particle perpendicular to its velocity vector cannot change its direction of motion without increasing its kinetic energy. If the particle's kinetic energy is to remain unchanged, the impulsive force must have a component in the direction opposite to the direction of motion. This situation is also realized in the case of a continuous force acting perpendicular to the velocity vector of the particle, when the particle's motion is viewed as a sequence of infinitesimal steps.
\end{abstract}

\section{Keywords}

Work, Normal Force, Kinetic Energy, Speed

\section{Introduction}

According to the work-energy theorem, the power delivered to a particle by the net force on it is equal to the time rate of change of its kinetic energy [1] [2] [3],

$$
\frac{\mathrm{d} T}{\mathrm{~d} t}=\boldsymbol{F} \cdot \boldsymbol{v}
$$

where $\boldsymbol{F}$ is the net force acting on the particle. As a result, if the net force has a component in the direction of motion, the kinetic energy of the particle increases. Conversely, if the net force has a component in the opposite direction of motion, the kinetic energy of the particle decreases. In each case, the normal component of the net force changes the direction of motion of the particle. If the net force is strictly normal to the velocity vector of the particle, it only changes the direction of motion of the particle without affecting its kinetic energy and speed. To this end, it is stated that normal force does not do work [4] [5] [6].

While developing a computer simulation, a moving particle was successively impacted perpendicular to its direction of motion to generate a circular path. During the simulation, it was noticed that the speed of the particle increased after each impact even though the impulsive force was perpendicular to the instantaneous velocity of the particle. This indicated that in this case the normal 
force did in fact do work on the particle. Although this is not a significant discovery by any scale, it warrants some discussion and should be brought to the attention of the computational science community.

In this article, a fairly detailed analysis of the dynamics of a particle subjected to a net force acting normal to its direction of motion is presented. The case of an impulsive force as well as the case of a continuous force, such as the magnetic force on a moving charged particle, or gravitational force on a circularly orbiting planet or satellite, is considered. The question of the change of kinetic energy and speed of the particle in each case is addressed, and it is shown that unless one is careful enough during the simulation, the normal force can do work on the particle and change its kinetic energy and speed.

To prevent the kinetic energy and speed from changing, one can always rescale the speed of the particle after each application of the normal force. This, however, is not a common practice in computational physics and, as a result, the kinetic energy and speed of the particle increase over time and error accumulates [7]. In this article, a different algorithm is suggested to avoid this problem. The algorithm is simple and more efficient than rescaling the speed in every step of the calculations.

\section{The Impulsive Force}

Let us begin by considering a particle of mass $m$ moving with a constant velocity $V$ along the $x$ axis. At a certain time, an impulsive force $F$ acts on the particle in the $y$ direction for a short time interval $\delta t$. The velocity of the particle after the action of the force is given by

$$
\boldsymbol{v}^{\prime}=\boldsymbol{v}+\frac{\boldsymbol{F} \delta t}{m}=v \hat{i}+\frac{F \delta t}{m} \hat{j}
$$

which simply indicates that the speed of the particle has increased to

$$
v^{\prime}=\sqrt{v^{2}+\left(\frac{F \delta t}{m}\right)^{2}}>v
$$

This difficulty seems to result from the finite duration of the time interval $\delta t$ over which the particle changes its direction of motion slightly, causing the force to gain a component in the direction of motion during this time interval. However, the problem can not be resolved even if the time interval is shrunk to zero and the force is reduced to a Dirac delta function. To see this, consider an impulsive force given by

$$
\boldsymbol{F}(t)=p \delta\left(t-t_{0}\right) \hat{j}
$$

where here $\delta$ denotes the Dirac delta function, and the force acts on the particle at time $t_{0}$. In this equation, $p$ is a constant having the units of momentum (as the delta function has the units of reciprocal time). The velocity of the particle after the action of the force is given by

$$
\boldsymbol{v}^{\prime}=v \hat{i}+\frac{p \hat{j}}{m} \int_{t_{0}-\epsilon}^{t_{0}+\epsilon} \delta\left(t-t_{0}\right) \mathrm{d} t=v \hat{i}+\frac{p}{m} \hat{j}
$$


where, again, the speed of the particle increases. The conclusion is that it is impossible to change the direction of motion of a particle by an impulsive force acting normal to its velocity vector without increasing its speed and kinetic energy. If the speed of the particle is to remain constant and only the direction of motion is to change, the impulsive force must act somewhat "backward". To see this, consider a particle of mass $m$ which is initially moving along the $x$ axis and is subjected to an impulsive force that acts on it for a short time interval $\delta t$ as it passes through the origin (Figure 1). The force acts at an angle $\alpha$ backward from normal to the direction of motion as shown. This force causes the $x$ component of velocity to decrease, but at the same time generates a $y$ component. The velocity of the particle after the action of the force is given by

$$
\boldsymbol{v}^{\prime}=\left(v-\frac{F \delta t}{m} \sin \alpha\right) \hat{i}+\left(\frac{F \delta t}{m} \cos \alpha\right) \hat{j}
$$

Now choose $\alpha$ so that the speed of the particle remains constant, i.e.,

$$
\left(v-\frac{F \delta t}{m} \sin \alpha\right)^{2}+\left(\frac{F \delta t}{m} \cos \alpha\right)^{2}=v^{2}
$$

This gives

$$
\sin \alpha=\frac{F \delta t}{2 m v}
$$

Therefore, if the particle is acted upon by an impulsive force that is not normal to its velocity vector, but makes a backward angle $\alpha$ given by Equation (8), its speed and the kinetic energy remain unchanged. This force changes the direction of motion of the particle only. It is interesting to note that the new direction of motion makes an angle $2 \alpha$ with the initial direction of motion. To see this, note that if we call this angle $\beta$, then from Equation (6) we have

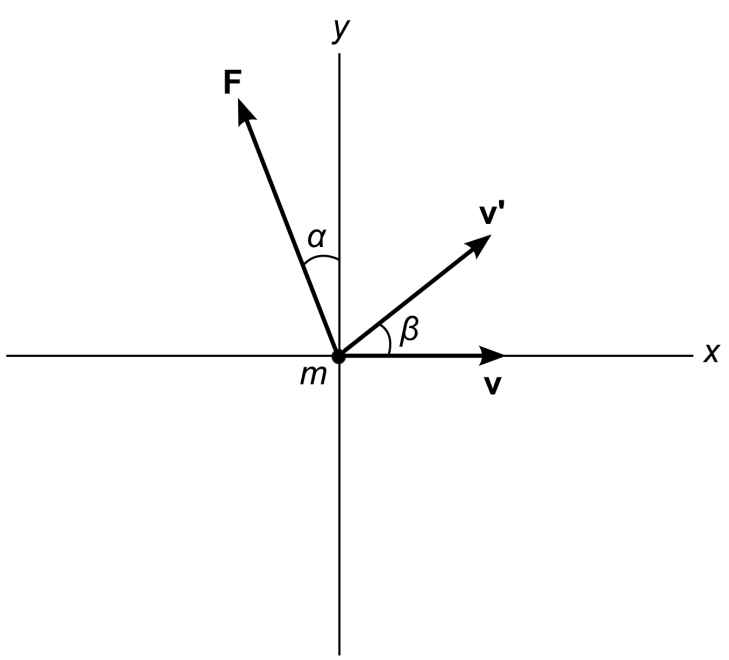

Figure 1. A particle of mass $m$ moving initially along the $x$ axis is subjected to an impulsive force $\boldsymbol{F}$ that acts on it during a short time period as the particle passes through the origin. $v$ and $v^{\prime}$ are the velocities of the particle before and after the impact, respectively. 


$$
\tan \beta=\frac{v_{y}^{\prime}}{v_{x}^{\prime}}=\frac{\frac{F \delta t}{m} \cos \alpha}{v-\frac{F \delta t}{m} \sin \alpha}
$$

Substituting for $F \delta t / m$ from Equation (8), reduces Equation (9) to

$$
\tan \beta=\frac{2 \sin \alpha \cos \alpha}{1-2 \sin ^{2} \alpha}=\frac{\sin 2 \alpha}{\cos 2 \alpha}=\tan 2 \alpha
$$

which proves the statement. Furthermore, this result, together with Equations (2) and (8), reveal that the new direction of motion is the same as what it would have been had the force acted perpendicular to the direction of motion.

\section{The Continuous Force}

Now consider the case of a continuous force that acts on a particle perpendicular to its velocity vector at all times. Figure 2 shows the path of such a particle during an infinitesimal time interval $\delta t$ starting when the particle is at the origin. The $x$ axis is chosen to coincide with the velocity vector of the particle at the beginning of this time interval. The force does not need to have a constant magnitude, however, if we choose $\delta t$ small enough then the magnitude of the force can be considered to be constant. The velocity vector of the particle at the end of the time interval is $\boldsymbol{v}^{\prime}$, which makes an angle $\delta \theta$ with the $x$ axis.

Let us use the force $\boldsymbol{F}$ at the beginning of the infinitesimal interval to calculate the speed $v^{\prime}$ at the end of the interval (Figure 2). This gives

$$
\boldsymbol{v}^{\prime}=v \hat{i}+\frac{F \delta t}{m} \hat{j}
$$

from which we obtain

$$
v^{\prime}=v\left[1+\frac{1}{2}\left(\frac{F \delta t}{m v}\right)^{2}\right]
$$

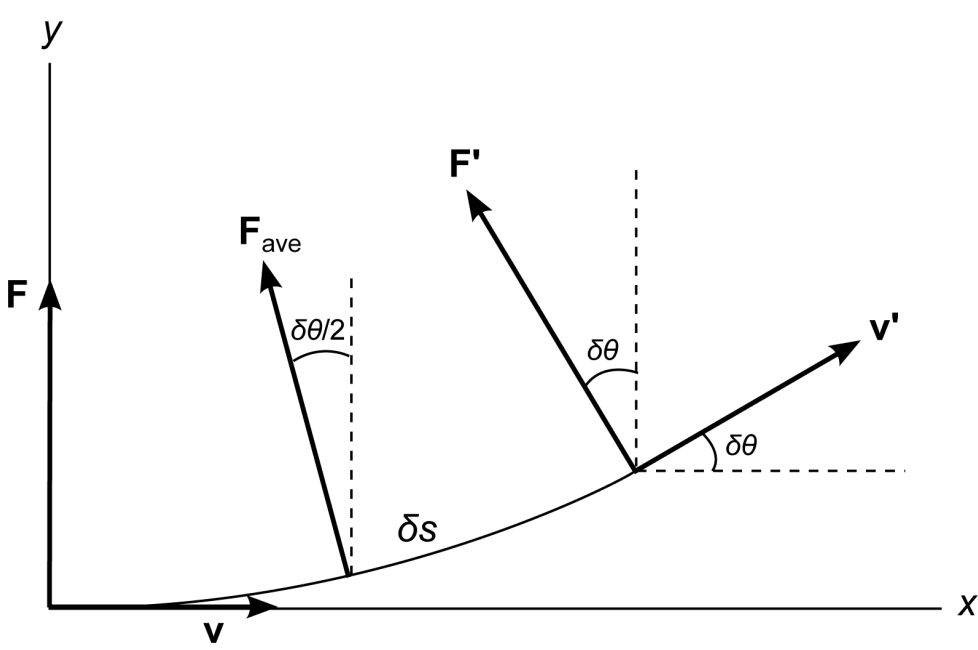

Figure 2. A normal force acting on a particle during an infinitesimal time interval. The coordinate system is chosen so that at the beginning of the time interval the particle is passing through the origin with velocity in the $x$ direction. 
where the last equality is written because $\delta t$ is infinitesimal and the higherorder terms are negligible. Since according to Equation (12), $\delta v=v^{\prime}-v$ is second-order in $\delta t$, integration over time gives a first-order increase in the speed of the particle [8]. On the other hand, if we use the force $\boldsymbol{F}^{\prime}$ at the end of the interval to calculate $v^{\prime}$, we obtain

$$
\boldsymbol{v}^{\prime}=\left[v-\frac{F \sin (\delta \theta) \delta t}{m}\right] \hat{i}+\left[\frac{F \cos (\delta \theta) \delta t}{m}\right] \hat{j}
$$

which gives

$$
v^{\prime 2}=v^{2}+\left(\frac{F \delta t}{m}\right)^{2}-\frac{2 v F \sin (\delta \theta) \delta t}{m}
$$

However, since $\delta \theta$ and $\delta t$ are both infinitesimal quantities, we can write

$$
\delta \theta=\sin (\delta \theta)=\tan (\delta \theta)=\frac{v_{y}^{\prime}}{v_{x}^{\prime}}=\frac{F \delta t}{m v}
$$

where the last equality can be obtain either from Equation (11), or from Equation (13) by neglecting the second- and higher-order terms in $F \delta t / m v$. Therefore, Equation (14) reduces to

$$
v^{\prime}=v\left[1-\frac{1}{2}\left(\frac{F \delta t}{m v}\right)^{2}\right]
$$

where again because $\delta t$ is infinitesimal, the higher-order terms are negligible. In this case, integration over time gives a first-order decrease in the speed of the particle. Therefore, using the initial value of the force over each interval slightly increases the speed of the particle, whereas using the final value of the force slightly decreases it.

Consider now the average of the two forces $\boldsymbol{F}$ and $\boldsymbol{F}^{\prime}$ over the infinitesimal interval,

$$
\bar{F}_{x}=-\frac{F}{2} \sin (\delta \theta) \quad \text { and } \quad \bar{F}_{y}=\frac{F}{2}[1+\cos (\delta \theta)]
$$

Using this average force, the components of the velocity at the end of the time interval are given by

$$
v_{x}^{\prime}=v-\frac{F \sin (\delta \theta) \delta t}{2 m} \quad \text { and } \quad v_{y}^{\prime}=\frac{F[1+\cos (\delta \theta)] \delta t}{2 m}
$$

from which we find

$$
v^{\prime 2}=v^{2}+\frac{F^{2} \delta t^{2}}{2 m^{2}}[1+\cos (\delta \theta)]-\frac{v F \delta t}{m} \sin (\delta \theta)
$$

Now writing

$$
\cos (\delta \theta)=1-\frac{\delta \theta^{2}}{2 !}, \quad \sin (\delta \theta)=\delta \theta-\frac{\delta \theta^{3}}{3 !}, \quad \tan (\delta \theta)=\delta \theta+\frac{\delta \theta^{3}}{3}
$$

which together give

$$
\sin (\delta \theta)=\tan (\delta \theta)-\frac{\delta \theta^{3}}{2}
$$


and substituting for $\tan (\delta \theta)$ from Equation (15), which is also valid in this case as can be seen from Equation (18), reduces Equation (19) to

$$
v^{\prime 2}=v^{2}-\frac{F^{2} \delta t^{2}}{4 m^{2}} \delta \theta^{2}+\frac{v F \delta t}{2 m} \delta \theta^{3}
$$

Finally, substituting for $\delta \theta$ from Equation (15) gives

$$
v^{\prime}=v\left[1+\frac{1}{8}\left(\frac{F \delta t}{m v}\right)^{4}\right]
$$

where the terms higher than 4 th order have been neglected. Since $\delta v=v^{\prime}-v$ is fourth order in $\delta t$, integration over time gives a change in the speed that is third-order in $\delta t$ [8]. Consequently, the speed of the particle remains constant to a high order.

It is interesting to note that the angle that the average force in the infinitesimal interval makes with the $y$ axis is exactly $\delta \theta / 2$ (Figure 2). To see this, let us denote the angle between the average force and the $y$ axis by $\gamma$. Then from Equation (17) we have

$$
\tan \gamma=\frac{\sin (\delta \theta)}{1+\cos (\delta \theta)}=\tan \left(\frac{\delta \theta}{2}\right)
$$

which proves the statement. Furthermore, since $\delta \theta$ is infinitesimal, from Equation (15) we have

$$
\sin \left(\frac{\delta \theta}{2}\right)=\frac{F \delta t}{2 m v}
$$

which is the same result as Equation (8) for the backward angle in the case of the impulsive force. It should be noted, however, that Equation (8) is exact whereas Equation (25) is an approximation obtained for a continuous force under the assumption of infinitesimal $\delta t$.

To sum up, the motion of the particle under a continuous force that is always perpendicular to its velocity vector can be viewed as consisting of a succession of infinitesimal steps or intervals. At the beginning of each step, the particle is acted upon by a force that is not perpendicular to the direction of motion but slightly pointing backward by a backward angle that is given by Equation (8) or (25). As a result of the action of this force, the speed and the kinetic energy of the particle remain unchanged over the interval, but the direction of motion changes by an angle equal to twice the backward angle.

\section{Conclusions}

It is impossible to change the direction of motion of a particle by a normal impulsive force without changing its kinetic energy and speed. If the latter is to remain unchanged, the impulsive force must act at a backward angle from the normal to the direction of motion.

This situation is also realized in the case of a continuous normal force, where the dynamics of the particle can be viewed as a sequence of infinitesimal steps. At the beginning of each step an impulsive force acts on the particle according to 
the rule described above. This conserves the kinetic energy and speed of the particle to a high degree of accuracy and only changes its direction of motion. This algorithm is particularly useful when numerical calculations of dynamics of a particle under a continuously varying net normal force, such as a charged particle moving in a magnetic field or a particle moving under a central force field, are concerned. Since in all computer simulations and numerical calculations finite time intervals are involved, the above algorithm provides an efficient tool for achieving a high degree of constancy in the kinetic energy and speed of the particle. Simply using a normal force at the beginning or at the end of the finite intervals will result in an increase or decrease of the kinetic energy and speed of the particle, respectively.

Finally, as was pointed out in the Introduction section, one can always let the force act perpendicular to the direction of motion of the particle, then rescale its speed after the action of the force. However, this process is more involved and results in a less efficient algorithm. The goal of this article, however, is not to just find a way to keep the speed and kinetic energy of the particle constant. The objective here, among other things, is to show that when a force normal to the velocity of a particle acts on it in a time interval $\delta t$, it does work on it regardless of how short $\delta t$ is, unless $\delta t=0$.

\section{References}

[1] Goldstein, H. (1980) Classical Mechanics. 2nd Edition, Addison-Wesley, Reading, 31.

[2] Marion, J.B. (1970) Classical Mechanics. Academic Press, New York, 65.

[3] Fowles, G.R. and Cassiday, G.L. (1993) Analytical Mechanics. 5th Edition, Saunders, New York, 117.

[4] Edwards, H.W. (1933) Analytical and Vector Mechanics. McGraw-Hill, New York, 147.

[5] Taylor, J.R. (2005) Classical Mechanics. University Science Books, Sausalito, California, 115 .

[6] Plantenberg, K. and Hill, R. (2013) Conceptual Dynamics. Chapter 7, SDC Publications, Mission, Kansas, 19.

[7] Gould, H. and Tobochnik, J. (1996) An Introduction to Computer Simulation Methods. Addison-Wesley, New York, 70-71.

[8] Gould, H. and Tobochnik, J. (1996) An Introduction to Computer Simulation Methods. Addison-Wesley, New York, 121. 
Submit or recommend next manuscript to SCIRP and we will provide best service for you:

Accepting pre-submission inquiries through Email, Facebook, LinkedIn, Twitter, etc. A wide selection of journals (inclusive of 9 subjects, more than 200 journals)

Providing 24-hour high-quality service

User-friendly online submission system

Fair and swift peer-review system

Efficient typesetting and proofreading procedure

Display of the result of downloads and visits, as well as the number of cited articles Maximum dissemination of your research work

Submit your manuscript at: http://papersubmission.scirp.org/

Or contact jamp@scirp.org 